\title{
Caracterização dos fatores de produção e uso de modelos matemáticos para estimar sua influência sobre o consumo diário de ração e a conversão alimentar de suínos nas fases de crescimento e terminação
}

\author{
[Characterization of production factors and use of mathematical models to estimate their influence \\ on daily feed intake and feed conversion ratio of grow-finishing pigs] \\ D.S. Borges ${ }^{1}$, P.S. Agostini ${ }^{2}$, C.R. Pierozan ${ }^{1 *}$, C.P. Dias ${ }^{1}$, M.A. Callegari ${ }^{1}$, A.K. Novais ${ }^{1}$, \\ R K.S. Santos ${ }^{1}$, M. Pereira Junior ${ }^{1}$, J.B. Alves ${ }^{1}$, J. Gasa ${ }^{3}$, C.A. Silva ${ }^{4}$ \\ ${ }^{1}$ Aluno de pós-graduação - Universidade Estadual de Londrina - Londrina, PR \\ ${ }^{2}$ Estudiant de postgrau - Universitat Autònoma de Barcelona - Bellaterra, CAT \\ ${ }^{3}$ Department de Ciència Animal i dels Aliments -Universitat Autònoma de Barcelona - Bellaterra, CAT \\ ${ }^{4}$ Universidade Estadual de Londrina - Londrina, PR
}

\begin{abstract}
RESUMO
O objetivo deste estudo foi identificar e quantificar os fatores de produção presentes em granjas de crescimento e terminação (CT) de suínos, em um sistema cooperativo do estado do Paraná, Brasil, e determinar a influência desses fatores no consumo diário de ração (CDR) e na conversão alimentar (CA). Foram avaliados, entre os anos de 2010 e 2013, os históricos produtivos de 538 lotes de engorda, alojados em 175 granjas comerciais, totalizando aproximadamente 404.000 animais. Foram considerados 40 fatores de produção, relacionados ao manejo, à sanidade, às instalações e aos equipamentos, à nutrição, à genética e ao ambiente. Em baias com menos de 20 suínos, o CDR diminuiu $(\mathrm{P}<0,05)$ e a CA melhorou $(\mathrm{P}<0,01)$. Nas baias com comedouros basculantes (lineares), o CDR reduziu $(\mathrm{P}<0,01)$ e a CA melhorou $(\mathrm{P}=0,001)$. Em baias com lâmina d'água, o $\mathrm{CDR}$ aumentou $(\mathrm{P}<0,01)$ e a $\mathrm{CA}$ piorou $(\mathrm{P}<0,01)$. Lotes formados apenas por fêmeas apresentaram menor CDR $(\mathrm{P}<0,001)$ e melhor $\mathrm{CA}(\mathrm{P}<0,001)$, quando comparados aos lotes mistos. As variáveis selecionadas para os modelos finais explicaram cerca de $25 \%$ e $46 \%$ da variância total para CDR e para a CA, respectivamente. Os modelos constituem um instrumento de auxílio efetivo para tomadas de decisões técnicas dentro do setor suinícola.
\end{abstract}

Palavras-chave: alimentação, índices de produção, modelagem, produção de suínos

\begin{abstract}
The aim of this study was to identify and quantify the production factors present in growing and finishing $(G F)$ pig farms partners of a cooperative in Paraná State, Brazil, and to determine the influence of these factors on daily feed intake (DFI) and the feed conversion ratio (FCR). From 2010 to 2013, the historical production of 538 batches of fattening pigs in 175 commercial farms were analyzed, in an amount approximately 404,000 animals. A total of 40 production factors regarding the management, sanity, plant and equipment, nutrition, genetics and environment were taken into consideration. Pens with less than 20 pigs resulted in a reduction in DFI $(P<0.05)$ and an improved FCR $(P<0.01)$. In pens that used linear dump feeders, the DFI was less $(P<0.01)$ and the FCR was better $(P=0.001)$. Pens with shallow pools had higher DFI $(P<0.01)$ and worse FCR $(P<0.01)$. Batches of females had lower DFI $(P<0.001)$ and better FCR $(P<0.001)$ compared to mixed-sex batches. The factors used for the final models explained the $25 \%$ and $46 \%$ of the total variance for the DFI and FCR, respectively. The models consist in effective aid instruments for technical decision making in the swine industry.
\end{abstract}

Keywords: feeding, modeling, pig production, production indexes

Recebido em 4 de dezembro de 2016

Aceito em 10 de fevereiro de 2017

*Autor para correspondência (corresponding author)

E-mail: carlospierozan@ hotmail.com 


\section{INTRODUÇÃO}

Aproximadamente mais de $75 \%$ da suinocultura brasileira é integrada às agroindústrias e às cooperativas (Panorama..., 2015; Relatório..., 2015). Esse desenho organizacional se mostra menos instável e menos vulnerável economicamente para o produtor, promove a melhora da eficiência produtiva por meio da especialização do processo e torna o segmento mais competitivo com a utilização de mais tecnologias (nutrição, genética, sanidade), atendendo as exigências de mercado (Severo, 2007). Nesse cenário, a maioria dos integrados e cooperados no país desenvolvem as fases de crescimento/terminação (CT) (Daga et al., 2008), sendo responsáveis pela oferta de mais de 30 milhões de suínos por ano (Panorama..., 2015; Relatório..., 2015).

Embora sejam consideradas fases cuja condução se apresenta pouco exigente, as fases de CT são consideradas críticas em termos de custos, dado o peso econômico decorrente da alimentação. Considerando uma granja de ciclo completo, essas fases representam $62 \%$ do custo de produção, devido ao elevado consumo de ração pelos animais (Girotto e Santos Filho, 2000).

Apesar da importância econômica das fases de CT, são escassos os estudos em condições brasileiras para quantificar o real impacto financeiro e zootécnico dos vários fatores de produção e do efeito da correlação nesse período. Para a quantificação desses efeitos sobre os fatores de produção, têm-se desenvolvido modelos matemáticos que, se validados corretamente, tornam-se uma ferramenta eficiente para previsão da relação entre os parâmetros sobre um determinado índice produtivo (Silva et al., 2015).

Nesse sentido, objetivou-se com este trabalho identificar e quantificar os fatores de produção que podem influenciar nos resultados zootécnicos das granjas de CT ligadas ao cooperativismo no oeste do estado do Paraná, Brasil; além de determinar a influência desses fatores no consumo diário de ração e na conversão alimentar, utilizando-se modelos matemáticos como ferramenta nas tomadas de decisões técnicas demandadas no sistema de produção.

\section{MATERIAL E MÉTODOS}

Toda informação sobre os lotes avaliados foi proveniente de uma base de dados e, portanto, a aprovação pela Comissão de Ética no Uso de Animais não foi necessária, uma vez que não houve uso de animais no presente trabalho. Entre os anos de 2010 e 2013 foram avaliados os históricos produtivos de 538 lotes de engorda, de 175 granjas de CT comerciais, localizadas no oeste do estado do Paraná, vinculadas a um sistema cooperativo.

O modelo de trabalho seguiu as experiências desenvolvidas no trabalho de Agostini et al. (2013). O projeto foi elaborado seguindo duas etapas distintas. A primeira envolveu a escolha das variáveis de interesse zootécnico e dos fatores de produção a serem investigados, levando em consideração a experiência acumulada pela equipe proponente, técnicos das empresas participantes e, também, as referências de trabalhos técnicos e científicos publicados recentemente. Diferentemente do estudo de Agostini et al. (2013), foram coletados os dados pertencentes a todas as explorações integradas na empresa e com maior número de lotes por granja. Ademais, todas as granjas avaliadas produziram lotes de engorda em diferentes estações do ano.

A segunda etapa consistiu na aplicação de questionários que compreendiam informações gerais sobre manejo e sanidade dos animais e instalações da unidade, os quais foram obtidos no formato de planilhas digitais e papel, estabelecendo-se um modelo para a coleta das informações. Assim, foi criado um sistema que garantisse a representatividade dos dados coletados dentro da empresa participante. O lote foi considerado como unidade experimental, o qual foi definido como um grupo de leitões que saiu da fase de creche para entrar na unidade de CT, onde permaneceram até o abate. Todos os lotes foram manejados no sistema all-in all-out.

As variáveis foram definidas como independentes e dependentes. Foram definidas como variáveis independentes: peso de entrada (PE), peso de saída (PS) e duração do período de CT (DCT) (variáveis contínuas) (Tab. 1) e um conjunto de 40 fatores de produção (variáveis categóricas) (Tab. 2). O PE ( $\mathrm{kg}$ ) correspondeu ao 
peso médio dos animais na entrada da fase de CT, e o PS $(\mathrm{kg})$ correspondeu ao peso médio dos animais registrado no abatedouro. A DCT (dias) foi o período, em dias, em que os animais permaneceram na unidade de CT.

Tabela 1. Descrição dos valores das variáveis contínuas em suínos nas fases de crescimento e terminação

\begin{tabular}{lcccccc}
\hline Variáveis & $\begin{array}{c}\text { Número de } \\
\text { lotes }\end{array}$ & Média & $\begin{array}{c}\text { Desvio- } \\
\text { padrão }\end{array}$ & Mínimo & Mediana & Máximo \\
\hline Peso de entrada $(\mathrm{kg})$ & 538 & 23,01 & 1,16 & 19,96 & 23,08 & 27,56 \\
Peso de saída (kg) & 538 & 116,43 & 3,83 & 104,34 & 116,37 & 129,69 \\
Duração da engorda (dias) & 538 & 109,61 & 2,95 & 100 & 110 & 118 \\
Consumo diário ração & 538 & 2,10 & 0,08 & 1,86 & 2,11 & 2,36 \\
(kg/suíno) & 538 & 2,47 & 0,11 & 2,16 & 2,46 & 2,78 \\
Conversão alimentar $(\mathrm{kg} / \mathrm{kg})$ & & & & & & \\
\hline
\end{tabular}

Tabela 2. Descrição das variáveis categóricas e suas frequências (porcentagem em relação ao número total de lotes estudados, $\mathrm{n}=538$ )

\begin{tabular}{|c|c|}
\hline Variável & Percentual (\%) de lotes em cada categoria \\
\hline Agricultura próxima à granja & $\operatorname{Sim}(89,01 \%) ;$ não $(10,99 \%)$ \\
\hline Árvores ao redor dos barracões & $\operatorname{Sim}(97,58 \%) ;$ não $(2,42 \%)$ \\
\hline Bebedouro ${ }^{(1)}$ & Chupeta $(76,39 \%)$; taça $(23,61 \%)$ \\
\hline Caixa de retenção de dejetos & $\operatorname{Sim}(18,96 \%)$; não $(81,04 \%)$ \\
\hline Capacidade de animais na granja & $<500(6,15 \%) ; 500-1000(93,85 \%)$ \\
\hline Choque com antibióticos & $\operatorname{Sim}(1,47 \%) ;$ não $(98,53 \%)$ \\
\hline Comedouro ${ }^{(1)(2)}$ & Semiautomático cônico $(52,42 \%)$; outros $(47,58 \%)$ \\
\hline Composteira & $\operatorname{Sim}(100 \%)$ \\
\hline Doença de Glasser, Erisipela & $\operatorname{Sim}(100 \%)$ \\
\hline Esterqueira & $\operatorname{Sim}(100 \%)$ \\
\hline Exaustores & Não $(100 \%)$ \\
\hline Forma física da ração & Peletizada $(100 \%)$ \\
\hline Ileíte, pneumonia enzoótica, meningite & $\operatorname{Sim}(100 \%)$ \\
\hline Lâmina d'água nas baias ${ }^{(1)(2)}$ & $\operatorname{Sim}(60,78 \%) ;$ não $(39,22 \%)$ \\
\hline Lotes sexados & $\operatorname{Sim}(100 \%)$ \\
\hline Luz elétrica & $\operatorname{Sim}(100 \%)$ \\
\hline Mão de obra & Não familiar $(18,96 \%)$; familiar $(81,04 \%)$ \\
\hline Material das tubulações de água & Tubo PVC (100\%) \\
\hline Material de construção dos barracões & Alvenaria $(74,91 \%)$; madeira e misto $(25,09 \%)$ \\
\hline Material de separação entre baias & Alvenaria $(98,52 \%)$; misto $(1,48 \%)$ \\
\hline Material do piso & Concreto $(100 \%)$ \\
\hline Material do telhado & Argila $(96,34 \%) ;$ amianto/zinco $(3,66 \%)$ \\
\hline Número de barracões & $\operatorname{Um}(50,93 \%)$; dois ou mais $(49,07 \%)$ \\
\hline Número de genéticas empregadas & Quatro $(100 \%)$ \\
\hline Número de rações utilizadas & Cinco $(100 \%)$ \\
\hline Número de suínos por baia ${ }^{(1)(2)}$ & $<20(68,96 \%) ;>20(31,04 \%)$ \\
\hline Origem da água & Poço/mina $(60,41 \%)$; rede $(39,59 \%)$ \\
\hline Origem dos suínos & Unidades produtoras de leitões (UPLs) $(100 \%)$ \\
\hline Posição da granja em relação ao nascer/pôr do sol & Norte/sul $(44,28 \%) ;$ leste/oeste $(55,72 \%)$ \\
\hline Programas utilizados & Ractopamina/imunocastração $(98,53 \%)$; ractopamina $(1,47 \%)$ \\
\hline Raças utilizadas & Large White/Landrace/Pietrain (100\%) \\
\hline Rações diferenciadas por sexo & Não $(100 \%)$ \\
\hline Reforma das instalações & $\operatorname{Sim}(15,02 \%)$; não $(84,98 \%)$ \\
\hline Semestre de entrada ${ }^{(1)(2)}$ & Verão/outono $(48,17 \%)$; inverno/primavera $(51,83 \%)$ \\
\hline Separação dos sexos nas baias & $\operatorname{Sim}(100 \%)$ \\
\hline $\operatorname{Sexo}^{(2)}$ & Castrados $(40,11 \%)$; fêmeas $(33,88 \%)$; misto $(26,01 \%)$ \\
\hline Tempo de construção ${ }^{(1)}$ & $<5$ anos $(50,56 \%) ;>5$ anos $(49,44 \%)$ \\
\hline Umidificadores/nebulizadores & $\operatorname{Sim}(54,28 \%) ;$ não $(45,72 \%)$ \\
\hline Ventiladores & Não $(100 \%)$ \\
\hline Via de uso dos antibióticos & Ração $(1,47 \%)$; água e ração $(98,53 \%)$ \\
\hline
\end{tabular}

${ }^{(1)}$ Variáveis selecionadas para o modelo final do consumo diário de ração (CDR). ${ }^{(2)}$ Variáveis selecionadas para o modelo final da conversão alimentar (CA). 
Dentro das variáveis dependentes, foram consideradas as variáveis contínuas: consumo diário de ração (CDR) e conversão alimentar (CA). O CDR (kg/suíno) foi calculado pela subtração da quantidade de ração consumida pelos lotes durante o período em CT da quantidade de sobra de ração nos silos no final do período. $\mathrm{O}$ resultado foi dividido pelo número total de animais do lote e, posteriormente, pelos dias de alojamento desses animais na fase de CT. A CA $(\mathrm{kg} / \mathrm{kg})$ foi calculada pelo total de ração fornecida a cada lote, dividido pela diferença entre o peso total de saída e o peso total de entrada.

As informações sobre as granjas foram coletadas e transferidas para uma planilha digital, utilizada como base para a realização das análises estatísticas, que foram realizadas usando-se o software estatístico SAS (Statistical..., 2009). Essas análises foram feitas em duas fases: análise exploratória e desenvolvimento de modelos, como em Maes et al. (2004), Oliveira et al. (2009) e Agostini et al. (2014).

$\mathrm{Na}$ análise exploratória, os dados foram submetidos à análise descritiva univariada e bivariada. As variáveis independentes categóricas foram submetidas a um estudo de frequência, usando-se o Proc FREQ do SAS. Para as variáveis contínuas, foram utilizadas medidas de tendência central (média e mediana) e de dispersão (desvio-padrão e amplitude), por meio do procedimento MEANS do SAS. As distribuições das variáveis contínuas foram avaliadas pelo procedimento UNIVARIATE do SAS.

Os modelos foram ajustados com base nas variáveis que foram codificadas na primeira fase, mediante regressão linear mista, com o efeito da granja e o do lote vinculados à granja sendo considerados como fatores aleatórios, usando-se o método de máxima verossimilhança restrita (REML) para a estimativa dos componentes de variância. A comparação do ajuste dos modelos finais foi realizada com base na proporção da variância explicada pelos diferentes modelos, empregando-se o coeficiente de determinação $\left(\mathrm{R}^{2}\right)$ como parâmetro.

Foi utilizado um modelo de regressão univariada, em que cada variável de previsão foi incluída como um único efeito fixo para cada variável dependente. As variáveis independentes que apresentaram $\mathrm{P} \leq 0,20$ foram selecionadas para utilização na análise multivariada, mediante o uso do procedimento MIXED do SAS.

Correlações de Pearson e Spearman foram realizadas entre as variáveis independentes no modelo multivariado para se evitar multicolinearidade entre as variáveis contínuas e os problemas de confusão entre as categóricas. Quando o coeficiente de correlação foi alto (valor absoluto $\geq 0,60$ ) entre duas variáveis, apenas uma foi usada na análise multivariada, sendo esta escolhida pela comparação dos valores de $\mathrm{P}$ na análise univariada, além de sua relevância biológica em relação à variável dependente. Posteriormente, todas as variáveis independentes selecionadas na análise univariada foram submetidas ao procedimento "stepwise", mantendo-se no modelo multivariado final todos os fatores que apresentaram $\mathrm{P}<0,05$. Testes para avaliação dos efeitos fixos foram baseados no teste $F$ com os graus de liberdade do denominador, sendo aproximados pelo procedimento Satterthwaite. Interações significativas $(\mathrm{P}<0,05)$ entre as variáveis no modelo multivariado foram testadas e incluídas. Após a montagem dos modelos para cada variável dependente (CDR e CA), os resíduos foram plotados contra os valores previstos para se verificar a homogeneidade das variâncias e a presença de outliers. Os fatores que apresentaram $\mathrm{P}<0,05$ no modelo final foram considerados estatisticamente significantes para uma dada variável dependente.

\section{RESULTADOS}

Houve grande prevalência de granjas com capacidade de alojamento para 500-1000 suínos $(93,85 \%)$, com a maioria das construções feitas em alvenaria $(74,91 \%)$, cobertas com telhas de argila $(96,34 \%)$ e com de piso de concreto nas baias em todas as unidades (Tab. 2).

Observou-se que a maioria das granjas apresenta baias pequenas, com capacidade para menos de 20 animais $(68,96 \%)$. Pouco mais da metade das granjas utiliza umidificadores e nebulizadores $(54,28 \%)$, bem como lâmina d'água nas baias $(60,78 \%)$, e a grande maioria possui árvores ao redor do barracão $(97,58 \%)$. 
A mão de obra utilizada nas granjas é predominantemente familiar $(81,04 \%)$, os comedouros tendem a ser semiautomáticos cônicos $(52,42 \%)$, e a maioria das granjas utiliza o programa de inclusão de ractopamina na ração associado à imunocastração $(98,53 \%)$.

O CDR por suíno por lote foi de $2,10 \pm 0,08 \mathrm{~kg}$ (variando de 1,86 a 2,36kg) (Tab. 1). Na regressão múltipla, as variáveis mantidas no modelo final que influenciaram significantemente o CDR foram: número de suínos por baia $(\mathrm{P}<0,05)$, tipo de comedouro $(\mathrm{P}<0,01)$, lâmina d'água nas baias $(\mathrm{P}<0,01)$, sexo $(\mathrm{P}<0,001)$ e peso de saída $(\mathrm{P}<0,001)$ (Tab. 3).
Em baias com capacidade para menos de 20 animais, o CDR diminuiu $0,026 \mathrm{~kg}$. Já nas baias que adotam comedouros basculantes/lineares, o CDR é reduzido em $0,028 \mathrm{~kg}$, comparado ao semiautomático cônico. Nas baias que apresentavam lâmina d'água, houve um acréscimo de $0,028 \mathrm{~kg}$ no CDR. Lotes de fêmeas apresentaram redução de $0,055 \mathrm{~kg}$ no $\mathrm{CDR}$ comparados com lotes mistos. Finalmente, a variável peso de saída exerceu um efeito de incremento do CDR. Para cada $1 \mathrm{~kg}$ de aumento no peso de saída, os suínos apresentaram acréscimo de $0,004 \mathrm{~kg}$ no consumo de ração diário.

Tabela 3. Modelo de regressão linear múltipla para o parâmetro consumo diário de ração (kg/suíno) de acordo com as variáveis relacionadas em 538 lotes de 175 granjas de suínos em fase de crescimento e terminação

\begin{tabular}{|c|c|c|c|c|}
\hline Efeito & Categoria & Estimativa (kg/suíno) & Erro-padrão & $\operatorname{Pr}>|t|$ \\
\hline Intercepto & - & 1,718 & 0,094 & $<0,001$ \\
\hline \multirow[t]{2}{*}{ Semestre de entrada } & Verão/outono & $-0,009$ & 0,006 & 0,103 \\
\hline & Inverno/primavera & 0 & - & - \\
\hline \multirow[t]{2}{*}{ Tempo construção } & $<5$ anos & $-0,009$ & 0,010 & 0,400 \\
\hline & $>5$ anos & 0 & - & - \\
\hline \multirow[t]{2}{*}{ Número de suínos por baia } & $<20$ & $-0,026$ & 0,011 & 0,019 \\
\hline & $20-40$ & 0 & - & - \\
\hline \multirow[t]{2}{*}{ Comedouro } & Outros (basculante/linear) & $-0,028$ & 0,009 & 0,002 \\
\hline & Semiautomático cônico & 0 & - & - \\
\hline \multirow[t]{2}{*}{ Bebedouro } & Chupeta & 0,018 & 0,010 & 0,086 \\
\hline & Taça & 0 & - & - \\
\hline \multirow[t]{2}{*}{ Lâmina d'água nas baias } & Sim & 0,028 & 0,009 & 0,003 \\
\hline & Não & 0 & - & - \\
\hline \multirow[t]{3}{*}{ Sexo } & Castrados & $-0,013$ & 0,007 & 0,068 \\
\hline & Fêmeas & $-0,055$ & 0,007 & $<0,001$ \\
\hline & Misto & 0 & - & - \\
\hline Peso de saída & - & 0,004 & 0,001 & $<0,001$ \\
\hline
\end{tabular}

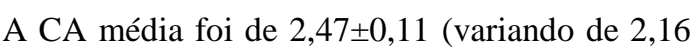
a 2,78) (Tab. 1). Na regressão múltipla, as variáveis selecionadas para o modelo final que interferiram significativamente na $\mathrm{CA}$ foram: número de suínos por baia $(\mathrm{P}<0,01)$, comedouro $(\mathrm{P}=0,001)$, lâmina d'água nas baias $(\mathrm{P}<0,01)$, sexo $(\mathrm{P}<0,001)$, peso de saída $(\mathrm{P}<0,001)$ e duração da engorda $(\mathrm{P}<0,001)$ (Tab. 4). Lotes alojados em baias com menos de 20 animais tiveram uma redução de cerca de 3,2 pontos na CA, comparados a lotes de suínos mantidos em baias com mais de 20 animais. Os comedouros basculantes resultaram em uma melhora de 3 pontos na CA comparados aos semiautomáticos cônicos. Animais provenientes de baias com lâmina d'água tiveram uma piora de 2,9 pontos na CA. Para a variável sexo, houve uma melhora de 3 pontos na CA para os lotes de machos castrados e de 7,1 pontos para os lotes de fêmeas, em relação aos lotes mistos. Com relação ao peso de saída, animais mais pesados ao abate resultaram em uma melhora da $\mathrm{CA}$, em que, para cada $1 \mathrm{~kg}$ de peso ganho, o índice mostra-se 1,7 ponto melhor, ao contrário da duração da engorda, a qual ocasiona piora de 1,2 ponto na CA para cada dia a mais de alojamento na unidade de CT. 
Tabela 4. Modelo de regressão linear múltipla para o parâmetro conversão alimentar $(\mathrm{kg} / \mathrm{kg})$ de acordo com as variáveis relacionadas em 538 lotes de 175 granjas de suínos em fase crescimento e terminação

\begin{tabular}{ccccc}
\hline Efeito & Categoria & Estimativa (kg/kg) & Erro-padrão & $\operatorname{Pr}>|\mathrm{t}|$ \\
\hline Intercepto & - & 3,136 & 0,150 & $<0,001$ \\
Semestre de entrada & Verão/outono & $-0,012$ & 0,011 & 0,273 \\
Número de suínos por baia & Inverno/primavera & 0 & - & - \\
& $<20$ & $-0,032$ & 0,011 & 0,006 \\
Comedouro & $20-40$ & 0 & - & - \\
& Outros (basculante/linear) & $-0,030$ & 0,009 & 0,001 \\
Lâmina d'água nas baias & Semiautomático cônico & 0 & - & - \\
& Sim & 0,029 & 0,009 & 0,003 \\
Sexo & Não & 0 & - & - \\
& Castrados & $-0,030$ & 0,009 & 0,007 \\
Peso de saída & Fêmeas & $-0,071$ & 0,009 & $<0,001$ \\
Duração da engorda & Misto & 0 & - & - \\
& - & $-0,017$ & 0,001 & $<0,001$ \\
\end{tabular}

As variáveis incluídas no modelo final para CDR explicaram $25 \%$ da variância encontrada para essa variável dependente. A proporção da variabilidade encontrada entre granjas relacionadas à cooperativa foi de 55,9\% e entre lotes relacionados a uma granja da cooperativa foi de $15,2 \%$ (Tab. 5).
Cerca de $46 \%$ da variância total encontrada para CA foram explicados pelas variáveis incluídas no modelo final. A proporção da variabilidade encontrada entre granjas relacionadas à cooperativa foi de $46 \%$ e entre lotes relacionados a uma granja da cooperativa foi de $45,5 \%$ (Tab.5).

Tabela 5. Estimativa dos parâmetros da variância nos modelos referentes ao consumo diário de ração e à conversão alimentar analisada a partir de 538 lotes de 175 granjas de suínos nas fases de crescimento e terminação

\begin{tabular}{ccc}
\hline & Consumo diário de ração & Conversão alimentar \\
\cline { 2 - 3 } & (Tab. 3) & (Tab. 4) \\
\hline R-quadrado do modelo & 0,25 & 0,46 \\
\% variabilidade explicada entre granjas (empresa) & 55,9 & 46,0 \\
\% variabilidade explicada entre lotes & 15,2 & 45,5 \\
\hline
\end{tabular}

\section{DISCUSSÃO}

No presente estudo, a influência das variáveis é explicada em $25 \%$ para o CDR e em $46 \%$ para a $\mathrm{CA}$, o que representa valores diferentes dos obtidos por Silva et al. (2015), que encontraram $41 \%$ para o CDR e $55 \%$ para a CA. Essa diferença entre as variáveis pode ser explicada pela subjetividade das informações, devido à ampla variabilidade dos fatores estudados, do período realizado e do local de estudo onde foram conduzidos os experimentos (Agostini et al., 2014).

Um aspecto observado neste estudo refere-se à variabilidade explicada entre granjas e entre lotes dentro da cooperativa, com os modelos multivariados de CDR e CA. Cerca de 15,2\% e
45,5\% da variabilidade da CDR e CA, respectivamente, foram explicados entre lotes da mesma granja. Essa maior proporção da variabilidade explicada entre lotes para CA é devido à inclusão da variável duração da engorda (uma variável tomada por lote, e não por exploração) no modelo. Essa variável não foi incluída no modelo para CDR.

Lotes de animais mantidos em baias com capacidade inferior a vinte animais apresentaram menor CDR e melhor CA. Resultados semelhantes foram encontrados por Ferguson et al. (2001), que constataram que animais mantidos individualmente tiveram consumo de ração diário superior ao daqueles mantidos em grupos de sete ou 13 animais por baia. Os animais precisam de condições que permitam 
que eles expressem seus comportamentos naturais. Sabendo-se que o bem-estar está relacionado com conforto físico e mental, baias com altas lotações podem interferir diretamente no desempenho e na sanidade, levando os animais a apresentarem maior estresse, resultado de um conjunto de respostas fisiológicas e comportamentais a uma ameaça (Veloni et al., 2013). Para Heck (2009), tanto a lotação quanto o tamanho do grupo afetam negativamente o desempenho dos animais. Grupos grandes de suínos são afetados por restrição de espaço mais rapidamente do que grupos pequenos. Em um estudo em que se avaliou o padrão de consumo de suínos mantidos em grupos de dois, quatro, oito e 12 animais por baia, não foi observada variação no desempenho, porém identificou-se que o padrão de consumo foi significativamente modificado, pois o número de visitas diminuiu e o tempo por visita ao comedouro aumentou com o número de animais por baia (Hyun e Ellis, 2002).

Quanto aos comedouros, os modelos basculantes sobressaíram-se aos semiautomáticos cônicos, pois reduziram o CDR e melhoraram a CA. É bom salientar a necessidade de ajustes nos comedouros semiautomáticos cônicos para melhora da CA e redução de desperdício, o qual pode chegar a $5 \%$, afetando a eficiência do lote e, por conseguinte, gerando prejuízo (Manzke et al., 2012). Ao compararem três regulagens distintas, das cinco disponíveis, em um modelo de comedouro automático com ração seca, sendo a primeira geradora de $80 \%$ de cobertura com ração, a terceira, 55\%, e a quinta, 15\%, Goodband et al. (2008) obtiveram resultados de que com $80 \%$ de cobertura com ração houve um ganho de peso similar à cobertura de $15 \%$, porém houve aumento de custos em virtude do maior desperdício. Já a cobertura de $55 \%$ não influenciou negativamente a CA e o custo total com ração. Em qualquer modelo de comedouro, é necessário levar em consideração o espaço linear de comedouro, visto que animais que sofrem privação de ração tendem a ter pior ganho de peso e CDR devido ao comportamento agressivo com maior competição para chegar ao comedouro (Heck, 2009; Manzke et al., 2012).

Quanto à presença de lâmina d'água nas baias, houve piora de 2,9 pontos na CA e aumento de 0,028kg no CDR em baias que dispunham de tal recurso. Esses resultados foram semelhantes aos encontrados por Moreira et al. (2003) com animais na fase de crescimento, em que aqueles mantidos em baias com lâmina d'água tiveram um aumento no CDR de $0,2 \mathrm{~kg}$ comparados aos mantidos em baias com piso compacto. No entanto, não se obteve diferença significativa para conversão alimentar. Já na fase de terminação, não houve diferença significativa para ambos os parâmetros. Um maior CDR pode ser explicado por um aumento do conforto térmico dos animais alojados em baias com presença de lâmina d'água, devido à maior facilidade em dissipar o calor produzido. É necessário, portanto, um controle da qualidade do ar nas instalações, um manejo adequado da lâmina d'água para não expor esses animais a maiores riscos de saúde, devido a uma maior concentração de gases nas instalações (Pandorfi et al., 2007).

Outra observação a ser colocada é que, devido à predominância de mão de obra familiar nas granjas, baias com lâmina d'água tendem a facilitar o manejo dos dejetos e a manter o ambiente e os animais mais limpos, fato que pode exercer efeito sobre o consumo.

Com relação ao fator sexo, lotes formados apenas por fêmeas tiveram redução no CDR $(0,05 \mathrm{~kg})$ e melhora na CA $(7,1$ pontos $)$ em relação aos lotes mistos. Resultados semelhantes foram encontrados por Barbosa et al. (2002), os quais observaram que machos castrados consumiram mais ração que as fêmeas, sem diferença significativa no ganho de peso médio diário, apontando que há uma melhor conversão de energia líquida para as fêmeas em comparação aos machos castrados.

A influência dos fatores identificados sobre os parâmetros CDR e CA nas fases de CT atua de forma ativa na produção, servindo como um termômetro para aferição dos resultados zootécnicos, tanto positiva quanto negativamente, além de sofrer alterações devido às rápidas mudanças que acontecem no sistema. Nesse sentido, devem ser considerados os custos, os investimentos demandados e a dinâmica do processo, tendo em vista que o modelo não é estático e que há a necessidade de atualizações frequentes dos parâmetros a serem analisados e da interferência desses sobre os índices produtivos. 


\section{CONCLUSÕES}

Os modelos matemáticos apontam efeitos quantitativos de alguns fatores de produção (manejo, instalações, equipamentos e sexo dos animais) sobre as variáveis consumo diário de ração (número de suínos por baia, comedouros, presença de lâmina d'água nas baias, sexo dos animais e peso de saída da engorda) e conversão alimentar (número de suínos por baia, comedouros, presença de lâmina d'água nas baias, sexo dos animais, peso de saída dos animais e duração da engorda). Com as frequentes mudanças demandadas nesse setor, objetivando a melhoria dos índices de produção, os modelos matemáticos devem ser atualizados e validados regularmente para garantirem a veracidade dos efeitos sobre os parâmetros de desempenho estudados e, assim, serem utilizados como um instrumento de auxílio efetivo para tomadas de decisões técnicas dentro do setor.

\section{AGRADECIMENTOS}

À Cooperativa participante, pela disponibilização dos dados e pela abertura das granjas; e ao Ministerio de Educación, Cultura y Deporte (AGL 2011-29960), pelo apoio financeiro.

\section{REFERÊNCIAS}

AGOSTINI, P.S.; FAHEY, A.G.; MANZANILLA, E.G. et al. Management factors affecting mortality, feed intake and feed conversion ratio of grow-finishing pigs. Animal, v.8, p.1312-1318, 2014.

AGOSTINI, P.S.; GASA, J.; MANZANILLA, E.G. et al. Descriptive study of production factors affecting performance traits in growingfinishing pigs in Spain. Span. J. Agric. Res., v.11, p.371-381, 2013.

BARBOSA, H.C.A.; VIEIRA, A.A.; TEIXEIRA, Z.S. et al. Desempenho de suínos em terminação alimentados com diferentes níveis de restrição alimentar e de energia na dieta. Rev. Bras. Ciênc. Vet., v.9, p.147-153, 2002.

DAGA, J.; CAMPOS, A.T.; FEIDEN, A. et al. Análise da adequação ambiental e manejo dos dejetos de instalações para suinocultura em propriedades na região oeste do Paraná. Eng. Agríc., v.27, p.1-11, 2008.
FERGUSON, N.S.; LAVERS, G.A.; GOUS, R.M. The effect of stocking density on the responses of growing pigs to dietary lysine. Anim. Sci., v.73, p.459-469, 2001.

GIROTTO, A.F.; SANTOS FILHO, J.J. Custo de produção de suínos. Concórdia: Embrapa/CNPSA, 2000. 36p. (Documentos, 62).

GOODBAND, B.; TOKACH, M.; DRITZ, S. et al. Feeding and feeder management influences on feed efficiency. In: ALLEN, D. LEMAN SWINE CONFERENCE, 2008, Minneapolis. Proceedings... Minneapolis: The University of Minnesota, 2008. p.20-27.

HECK, A. Fatores que influenciam o desenvolvimento dos leitões na recria e terminação. Acta Sci. Vet., v.37, Sup., p.S211S218, 2009.

HYUN, Y.; ELLIS, M. Effect of group size and type feeder on growth performance and feeding patterns in finishing pigs. J. Anim. Sci., v.80, p.568-574, 2002.

MAES, D.G.D.; DUCHATEAU, L.; LARRIESTRA, A. et al. Risk factors for mortality in grow-finishing pigs in Belgium. $J$. Vet. Med. B Infect. Dis. Vet. Public Health, v.51, p.321-326, 2004.

MANZKE, N.E.; DALLA COSTA, O.A.; LIMA, G.J.M.M. Atualidades e desafios nas fases de crescimento e terminação: 1) sistemas de alimentação. In: SEMINÁRIO INTERNACIONAL DE AVES E SUÍNOS AVESUI, 11., 2012, Florianópolis. Anais... Florianópolis: Gessulli, 2012.11p.

MOREIRA, I.; PAIANO, D.; OLIVEIRA, G.C. et al. Desempenho e características de carcaças de suínos $(33-84 \mathrm{~kg})$ criados em baias de piso compacto ou com lâmina d'água. Rev. Bras. Zootec., v.32, p.132-139, 2003.

OLIVEIRA, J.; YUS, E.; GUITIÁN, F.J. Effects of management, environmental and temporal factors on mortality and feed consumption in integrated swine fattening farms. Livest. Sci., v.123, p.221-229, 2009.

PANDORFI, H.; SILVA, I.J.O.; GUISELINI, C.; PIEDADE, S.M.S. Uso da lógica fuzzy na caracterização do ambiente produtivo para matrizes gestantes. Eng. Agríc., v.27, p.83-92, 2007. 
PANORAMA setorial da suinocultura: dossiê detalhado do setor suinícola. [Brasília]: ABCS, 2015. Disponível em: <http://issuu.com/ revistaabcs/docs/panorama_setorial>. Acessado em: 11 jan. 2016.

RELATÓRIO anual. [Brasília]: ABPA, 2015. Disponível em: <http://abpa-br.com.br/files/ RelatorioAnual_UBABEF_2015_DIGITAL.pdf> . Acessado em: 11 jan. 2016.

SEVERO, L.S. Evolução da sustentabilidade no processo produtivo de suínos da cooperativa de suinocultores de Encantado Ltda - COSUEL. 2007. 175f. Dissertação (Mestrado em Administração) - Universidade Federal do Rio Grande do Sul, Porto Alegre, RS.
SILVA, C.A.; AGOSTINI, P.S.; GASA, J. Uso de modelos matemáticos para analisar a influência de fatores de produção sobre a mortalidade e desempenho de suínos de terminação. In: BARCELLOS, D.E.; BORTOLOZZO, F.P.; WENTZ, I.; BERNARDI, M.L. (Eds.). Avanços em sanidade, produção e reprodução de suínos. Porto Alegre: Universidade Federal do Rio Grande do Sul, 2015. p.267-284.

STATISTICAL analysis system. Versão 9.2. Cary: SAS Institute, 2009.

VELONI, M.L.; PRADO, P.L.; ARSSUFFI, B.M. et al. Bem-estar animal aplicado nas criações de suínos e suas implicações na saúde dos rebanhos. Rev. Cient. Eletr. Med. Vet., v.21, n.1, 2013. 